\title{
Analysis of Bridge-type Distributed-compliance Mechanism
}

\author{
Lei-Jie Lai ${ }^{1}$ and Xiao-Qia Yin ${ }^{2}$ \\ 1College of Mechanical Engineering, Shanghai University of Engineering Science, Shanghai, 201620, China \\ ${ }^{2}$ School of Electronic, Information and Electrical Engineering, Shanghai Jiao Tong University, Shanghai, 200240, China
}

\begin{abstract}
This paper analyses a class of bridge-type distributed-compliance mechanism, which has better performances than traditional bridge-type mechanisms using notch flexure hinges. An analytical model for the displacement amplification ratio and input stiffness calculations of the bridge-type mechanism is established based on the stiffness matrix method. The finite element analysis results are then given to validate the correctness of the analytical model. The differences of the analytical results with respect to the finite element analysis results are less than $8 \%$, which demonstrate the high accuracy of the analytical model. The influences of the geometric parameters on the amplification ratio and input stiffness of the mechanism are also investigated using the analytical model to provide theoretical guidelines for the practical design.
\end{abstract}

\section{Introduction}

Recently, the piezoelectric actuator (PZT) is widely adopted due to its advantages of ultra-high resolution, fast response, high stiffness, compact size and high push force [1]. However, the relative small displacement produced by the PZT, which is about $10 \mu \mathrm{m} / \mathrm{cm}$ limits its applications of the larger working ranges. Therefore, in order to achieve larger output displacement, displacement amplification mechanisms are needed to amplify the stroke of PZT. Compared with the traditional lever-type mechanism, bridge-type flexure mechanism has the advantages of large amplification ratio, high resonant frequency and compact size, which becomes more and more popular.

In the previous literatures, many studies have been conducted to design and investigate bridge-type flexure mechanism. Lobontiu and Garcia formulated an analytical model for displacement and stiffness calculations of planar compliant mechanisms with singleaxis flexure hinges based on the Castigliano's second theorem [3]. Ma et al. derived the ideal displacement amplification ratio of bridge-type mechanism using kinematic theory, and also derived a theoretic displacement amplification ratio using the elastic beam theory and work-energy theorem [4]. Some other methods developed for the analytical models of the bridge-type mechanisms are also reported.

All of the bridge-type compliant mechanisms mentioned in the previous literatures are lumpedcompliance mechanisms, which use the notch flexure hinges. The existences of rigid bodies in the four arms of the lumped-compliance bridge-type mechanism have great weakness for the resonant frequency of the mechanism. Compared with the lumped-compliance mechanism, the distributed-compliance mechanism adopts four beam flexures to replace the notch flexure hinges and the rigid bodies, resulting in increased resonant frequency of the mechanism [5]. Moreover, the bending behavior of the beam flexure under the piezoelectric actuation allows for an acceptable distribution of the stresses in the distributed-compliance mechanism, which ensures a longer life time and better performances than traditional mechanical amplifiers in the dynamic applications. However, the mechanical properties of the bridge-type distributed-compliance mechanism still not been fully discovered. In the design stage, simple yet accurate enough analytical models are required to predict the performances of the mechanism and to further determine its architectural parameters according to the performance requirements.

In this paper, the mechanical properties and deformation characteristics of bridge-type distributedcompliance mechanism are deeply analyzed. An analytical model for the predictions of theoretical displacement amplification ratio and input stiffness is derived using the stiffness matrix method and validated by FEA. The comparisons between the analytical model and FEA results demonstrate the high accuracy of the analytical model. The influences of geometric and material parameters on the performances of the mechanism including the displacement amplification ratio and input stiffness are also well analyzed according to the analytical model.

\section{Analytical model}


The schematic of the bridge-type distributed-compliance mechanism with rhombic shell is depicted in Figure 1. The compliant mechanism mainly consists of four beam flexures that are connected in the way of bridge type.

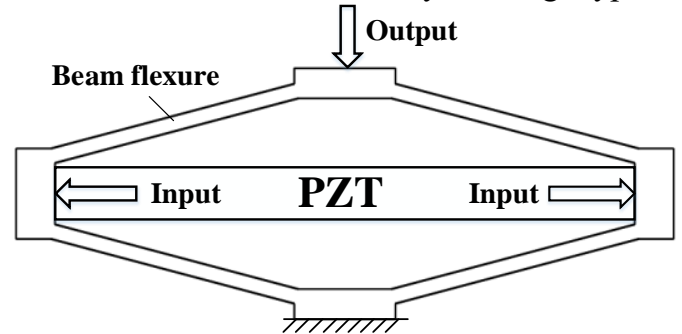

Figure 1. Schematic of bridge-type distributedcompliance

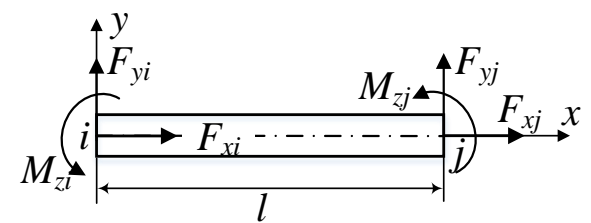

Figure 2. Model of a beam flexure mechanism

\subsection{Model of single beam flexure.}

The compliant mechanism contains four beam flexures, therefore, the stiffness characteristic of single beam flexure should be obtained first. As shown in Figure 2, two ends of the beam flexure are subjected to axial forces, bending moments and shear forces. In order to obtain the load-displacement relationship for the beam flexure, the right end of the beam is first fixed as shown in Figure 3(a). According to the elastic beam theory, the displacements of point $\mathrm{i}$ is

$$
\begin{aligned}
& u_{i}=\frac{F_{x i} l}{E A}, w_{i}=\frac{F_{y i} l^{3}}{3 E I}-\frac{M_{z i} l^{2}}{2 E I}, \\
& \theta_{i}=-\frac{F_{y i} l^{2}}{2 E I}+\frac{M_{z i} l}{E I}
\end{aligned}
$$

where $E$ is the Young's modulus of the material. $A$ and $I$ denote respectively the cross-sectional area and the second moment of inertia of the cross section about neutral axis. From the above three equations, the forces applied to point $i$ can be calculated as

$$
\begin{aligned}
& F_{x i}=\frac{E A}{l} u_{i}, F_{y i}=\frac{12 E I}{l^{3}} w_{i}+\frac{6 E I}{l^{2}} \theta_{i}, \\
& M_{z i}=\frac{6 E I}{l^{2}} w_{i}+\frac{4 E I}{l} \theta_{i}
\end{aligned}
$$

Based on the force equilibrium theory, the forces applied to point $j$ are

$$
\begin{aligned}
& F_{x j}=-\frac{E A}{l} u_{i}, F_{y j}=-\frac{12 E I}{l^{3}} w_{i}-\frac{6 E I}{l^{2}} \theta_{i}, \\
& M_{z j}=\frac{6 E I}{l^{2}} w_{i}+\frac{2 E I}{l} \theta_{i}
\end{aligned}
$$
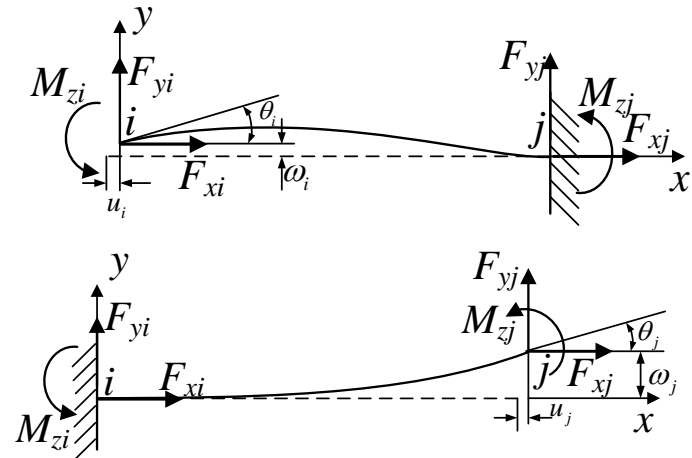

(a)

(b)

Figure 3. Two cases of beam structure. (a) Free-fixed. (b) Fixed-free

A similar procedure is applied by fixing the left end $i$ of the beam, as shown in Figure 3(b). In this case, the forces applied to both ends can be calculated as

$$
\begin{aligned}
& F_{x i}=-\frac{E A}{l} u_{j}, F_{y i}=-\frac{12 E I}{l^{3}} w_{j}+\frac{6 E I}{l^{2}} \theta_{j}, \\
& M_{z i}=-\frac{6 E I}{l^{2}} w_{j}+\frac{2 E I}{l} \theta_{j} \\
& F_{x j}=\frac{E A}{l} u_{j}, F_{y j}=\frac{12 E I}{l^{3}} w_{j}-\frac{6 E I}{l^{2}} \theta_{j}, \\
& M_{z j}=-\frac{6 E I}{l^{2}} w_{j}+\frac{4 E I}{l} \theta_{j}
\end{aligned}
$$

By superimposing the results of the above two cases, as shown in Figure 2, the stiffness equations can be written as

$$
\begin{aligned}
& F_{x i}=\frac{E A}{l} u_{i}-\frac{E A}{l} u_{j}, \\
& F_{y i}=\frac{12 E I}{l^{3}} w_{i}+\frac{6 E I}{l^{2}} \theta_{i}-\frac{12 E I}{l^{3}} w_{j}+\frac{6 E I}{l^{2}} \theta_{j} \\
& M_{z i}=\frac{6 E I}{l^{2}} w_{i}+\frac{4 E I}{l} \theta_{i}-\frac{6 E I}{l^{2}} w_{j}+\frac{2 E I}{l} \theta_{j} \\
& F_{x j}=-\frac{E A}{l} u_{i}+\frac{E A}{l} u_{j}, \\
& F_{y j}=-\frac{12 E I}{l^{3}} w_{i}-\frac{6 E I}{l^{2}} \theta_{i}+\frac{12 E I}{l^{3}} w_{j}-\frac{6 E I}{l^{2}} \theta_{j} \\
& M_{z j}=\frac{6 E I}{l^{2}} w_{i}+\frac{2 E I}{l} \theta_{i}-\frac{6 E I}{l^{2}} w_{j}+\frac{4 E I}{l} \theta_{j}
\end{aligned}
$$

\subsection{Displacement amplification ratio and input stiffness.}

Because of the symmetrical structure of the bridge-type mechanism, only a quarter model of the mechanism shown in Figure 4 needs to be analyzed. 


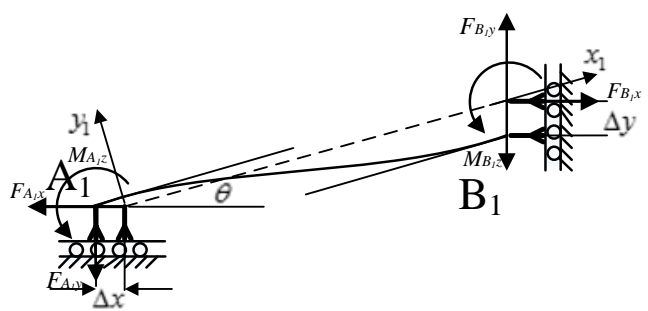

Figure 4. The quarter model of the bridge-type mechanism.

Because of the symmetrical structure of the mechanism, the orientations of the two rigid bodies are unchanged during the deformation process of the mechanism. According to the Euler-Bernoulli beam theory, the cross-sectional plane of deformed beam is still perpendicular to the deformed axis, the variations of rotation angles at Point $\mathrm{A}_{1}$ and Point $\mathrm{B}_{1}$ are both equal to zero. Therefore, the displacements at the Point $A_{1}$ and Point $\mathrm{B}_{1}$ in the $\mathrm{x}_{1}$ - and $\mathrm{y}_{1}$ - directions can be obtained:

$$
\begin{aligned}
& u_{A_{1}}=-\Delta x \cos \theta, w_{A_{1}}=\Delta x \sin \theta, \theta_{A_{1}}=0, \\
& u_{B_{1}}=-\Delta y \sin \theta, w_{B_{1}}=-\Delta y \cos \theta, \theta_{B_{1}}=0
\end{aligned}
$$

The forces applied to Point $A_{1}$ and Point $B_{1}$ in the $x_{1}$ and $\mathrm{y}_{1}$ - directions can be calculated as:

$$
\begin{aligned}
& F_{A x_{1}}=-F_{A x} \cos \theta-F_{A y} \sin \theta \\
& F_{A y_{1}}=F_{A x} \sin \theta-F_{A y} \cos \theta
\end{aligned}
$$

Then, the displacement amplification ratio and input stiffness can be calculated. The displacement amplification ratio and input stiffness without the consideration of the external force is

$$
\begin{aligned}
& R_{a m p}=\frac{\sin \theta \cos \theta\left(l^{2}-t^{2}\right)}{\left(t^{2} \cos ^{2} \theta+l^{2} \sin ^{2} \theta\right)}, \\
& K_{\mathrm{in}}=\frac{2 E b t^{3}}{l\left(t^{2} \cos ^{2} \theta+l^{2} \sin ^{2} \theta\right)}
\end{aligned}
$$

\section{Finite element analysis validation}

In this section, the finite element analysis (FEA) is performed to validate the analytical model formulated for evaluating the performances of this compliant mechanism. Three samples with different material and geometrical parameters and applied force conditions were set for the comparisons with FEA results, as listed in Table 1. The displacement amplification ratio and input stiffness calculated from the analytical model and the FEA model are listed in Table 2. The relative differences between the analytical results and FEA results of the three samples are all less than $8 \%$, which indicates that the two results are in good agreement.

Table 1: Parameters of different samples.

\begin{tabular}{|c|c|c|c|c|c|c|c|c|}
\hline Sample & $E(\mathrm{GPa})$ & $v$ & $l(\mathrm{~mm})$ & $b(\mathrm{~mm})$ & $t(\mathrm{~mm})$ & $\theta\left(^{\circ}\right)$ & $F_{I}(\mathrm{~N})$ & $F_{O}(\mathrm{~N})$ \\
\hline 1 & 71 & 0.33 & 48 & 10 & 1.8 & 12.5 & 100 & 20 \\
\hline 2 & 71 & 0.33 & 40 & 8 & 2 & 9 & 250 & 30 \\
\hline 3 & 200 & 0.3 & 50 & 12 & 2.2 & 8 & 200 & 50 \\
\hline
\end{tabular}

Table 2: Comparisons between the FEA and analytical results.

\begin{tabular}{|c|c|c|c|c|}
\hline \multirow{2}{*}{ Sample } & & $\begin{array}{c}\text { Analytical } \\
\text { model }\end{array}$ & $\begin{array}{c}\text { FEA } \\
\text { model }\end{array}$ & $\begin{array}{c}\text { Difference } \\
(\%)\end{array}$ \\
\hline \multirow{2}{*}{1} & $R_{a m p}$ & 3.39 & 3.41 & 0.6 \\
\cline { 2 - 5 } & $K_{\text {in }}(\mathrm{N} / \mu \mathrm{m})$ & 12.51 & 11.88 & 5.3 \\
\hline \multirow{2}{*}{2} & $R_{a m p}$ & 4.39 & 4.36 & 0.7 \\
\cline { 2 - 5 } & $K_{\text {in }}(\mathrm{N} / \mu \mathrm{m})$ & 16.87 & 15.61 & 8 \\
\hline \multirow{2}{*}{3} & $R_{a m p}$ & 8.20 & 8.08 & 1.48 \\
\cline { 2 - 5 } & $K_{\text {in }}(\mathrm{N} / \mu \mathrm{m})$ & 31.16 & 31.4 & 0.8 \\
\hline
\end{tabular}

\section{Influences of geometric parameters}

The analytical model is established to analyze the influences of the geometric parameters on the displacement amplification ratio and input stiffness. $l, b, t$ and $\theta$ are chosen as the designed geometric parameters of the mechanism. Figures 5-8 show the geometric parameters' influences on the displacement amplification ratio and input stiffness. It can be seen that the four geometric parameters have significant effects on the displacement amplification ratio and input stiffness:

$b$ has no influence on the amplification ratio, which increases slightly while $l$ increases and $t$ decreases. The variation rate of Ramp decreases gradually with the increase of $l$.
The amplification ratio Ramp has a maximum value when $\theta$ reaches a threshold value. When $\theta$ decreases continuously, the amplification ratio Ramp decreases sharply.

The input stiffness $K_{\text {in }}$ decreases with both $l$ and $\theta$ increasing. Both of the two curves decrease sharply first and the gently. $K_{\text {in }}$ also increases linearly with the increase of $b$. 


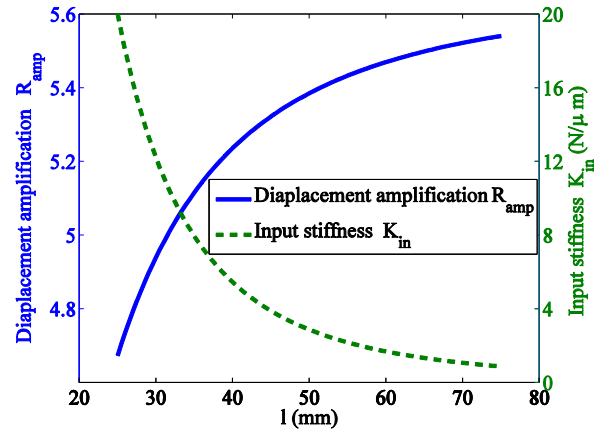

Figure 5: Influence of 1 on amplifier's performance.

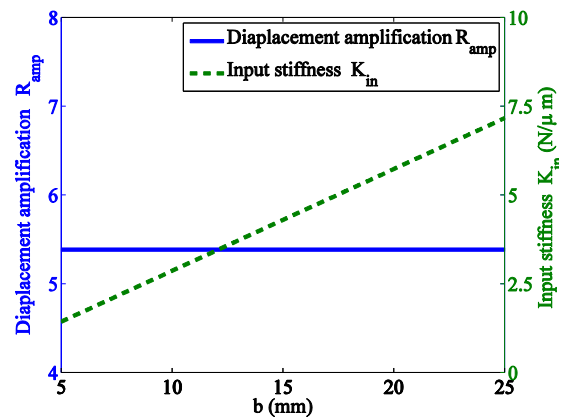

Figure 6: Influence of b on amplifier's performance.

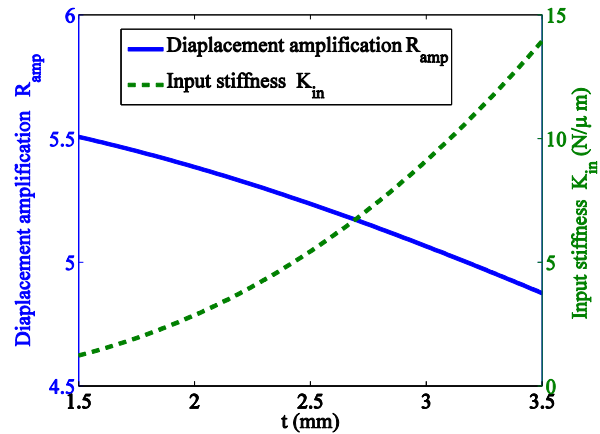

Figure 7: Influence of t on amplifier' performance.

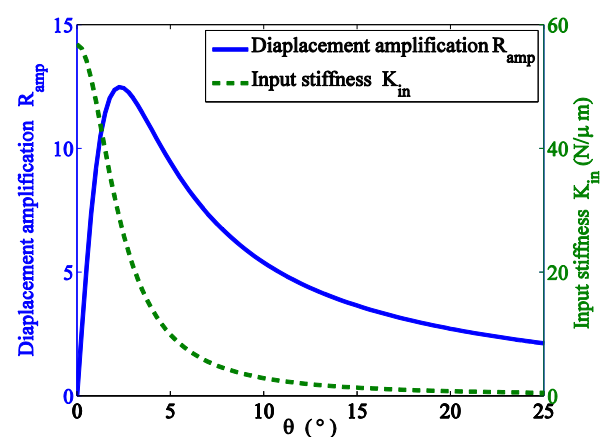

Figure 8: Influence of $\theta$ on amplifier's performance.

\section{Conclusions}

In this paper, a class of bridge-type distributedcompliance mechanism is analyzed. Due to distributed stresses and low mass, the bridge-type distributedcompliance mechanism has a longer life time and better performances than traditional mechanical amplifiers based on lever arm and flexure pivot. The stiffness matrix method is employed to derive analytical model to predict the displacement amplification ratio and input stiffness of the bridge-type mechanism. In order to verify the analytical model, the FEA simulations of the bridge-type mechanism are performed via the ANSYS Workbench. The comparisons show that the FEA and analytical results agree well with each other with the deviation of less than $8 \%$. The relationships between the dimensions of the bridge-type mechanism and its performances including the displacement amplification ratio and input stiffness are represented graphically, which provide a theoretical basis for the practical design.

\section{References}

1. Kim, Dongmin, et al. Optimal design of a flexure hinge-based XYZ atomic force microscopy scanner for minimizing Abbe errors. Review of Scientific Instruments 76.7 (2005): 073706 - 073706 -7.

2. Choi, Kee Bong, J. J. Lee, and S. Hata. A piezo-driven compliant stage with double mechanical amplification mechanisms arranged in parallel. Sensors \& Actuators A Physical 161. 1 (2010): 173-181.

3. Lobontiu, Nicolae, and E. Garcia. Analytical model of displacement amplification and stiffness optimization for a class of flexure-based compliant mechanisms. Computers \& Structures 81. 32 (2003): 2797-2810.

4. Ma, Hong Wen, et al. Analysis of the displacement amplification ratio of bridge-type flexure hinge. Sensors \& Actuators A Physical 132.2(2006):730-736.

5. Polit, Sebastian, and J. Dong. Development of a HighBandwidth XY Nanopositioning Stage for High-Rate Micro-/Nanomanufacturing.

IEEE/ASME Transactions on Mechatronics 16.4 (2011): 724-733. 